\title{
Nanoparticle Electric Propulsion: Experimental Results AIAA-2006-4803
}

\author{
Louis Musinski*, Thomas Liu ${ }^{\dagger}$, Brian Gilchrist*, Alec Gallimore ${ }^{\S}$, and Michael Keidar ${ }^{* *}$ \\ University of Michigan, Ann Arbor, MI, 48109
}

This paper presents experimental results concerning the nanoparticle Field Extraction Thruster (nanoFET) concept under development at the University of Michigan. The nanoFET concept offers an electric propulsion approach that can have a highly adjustable charge-to-mass ratio and electrostatic acceleration that potentially could span a specific impulse range from $\sim 100 \mathrm{~s}$ to $\sim 10,000 \mathrm{~s}$ and thrust power ranging from microwatts to many tens of kilowatts at high efficiency. Here, we report on experiments addressing particle charging, particle transport through an insulating liquid, particle extraction from a liquid, and Taylor cone formation. In addition, it has been shown that particles can be extracted from liquid using electric fields without the formation of Taylor cones or colloidal droplets. These experimental results have validated our initial theoretical models building confidence in the fundamental feasibility of the nanoFET concept.

\section{Nomenclature}

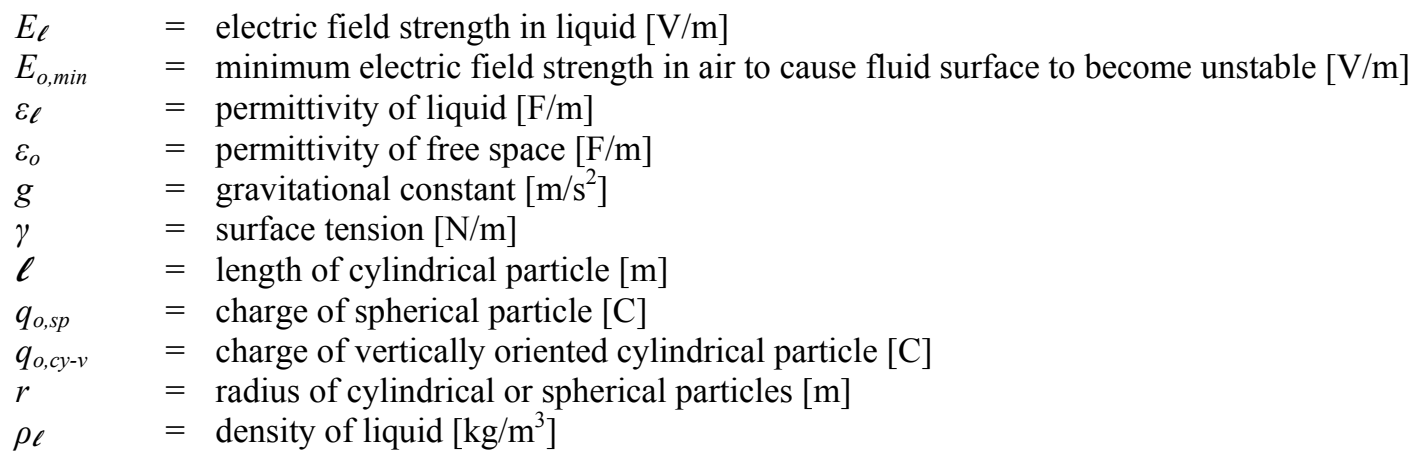

\footnotetext{
* Graduate Student, Electrical Engineering, louisdm@umich.edu, AIAA Student Member

† Graduate Student, Aerospace Engineering, liutm@umich.edu, AIAA Student Member

* Professor, Electrical Engineering \& Space Systems, gilchrst@umich.edu, AIAA Associate Fellow

${ }^{\S}$ Arthur F. Thurnau Professor, Aerospace Engineering \& Applied Physics, rasta@umich.edu, AIAA Associate Fellow

** Assistant Research Professor, Aerospace Engineering, keidar@umich.edu, Senior AIAA Member
} 


\section{Introduction}

A new concept (nanoFET) utilizing highly scalable MEMS (Micro-Electric-Mechanical Systems) technologies to create nanoparticle electrostatic propulsion is under development at the University of Michigan. ${ }^{1}$ At the core of the nanoparticle thruster, millions of MEMS based micron-sized vias, similar to what is shown in Figure 1, are used. A multi-layer grid establishes the critical electric fields to both extract and accelerate charged conducting nanoparticles (one example shown in Figure 2$)^{2}$ from the surface of

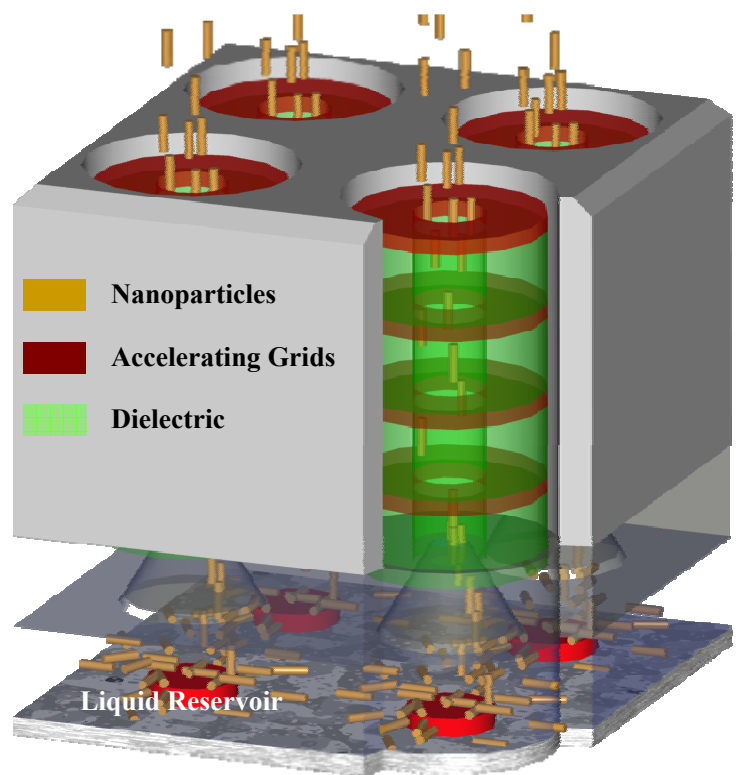

Figure 1: Four small vias shown extracting and accelerating nanoparticles.

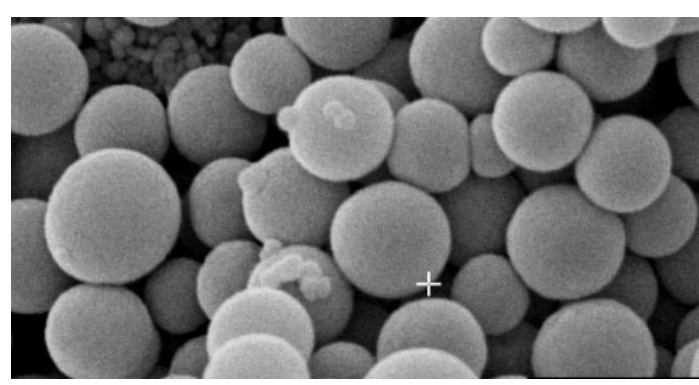

Figure 2: Example of a collection of unsorted spherical nanoparticles in the 100-nm diameter range. either an insulating or mildly conducting liquid used to transport these particles. These nanoparticles will likely have characteristic sizes ranging from $\sim 1 \mathrm{~nm}$ to $\sim 1 \mu \mathrm{m}$. We are investigating the nanoFET concept as a way to continuously tune the specific impulse and thrust over a very broad range at high efficiency. The specific impulse range will be principally limited by the size, shape, and density of the particles, as well as the potential that they fall through. It is expected that with the use of just three particle types, nanoFET can cover a specific impulse range from $100 \mathrm{~s}$ to $10,000 \mathrm{~s}$ while maintaining efficiencies greater than $90 \%$.

The nanoFET concept can be contrasted to that of utilizing field emission and electrostatic acceleration of ions, well known as Field Emission Electric Propulsion (FEEP). FEEP thrusters can produce specific impulses above 10,000 seconds at thrust efficiencies exceeding 90\% using melted metal liquid propellant such as indium. ${ }^{3,4,5,6}$ However, FEEPs use needle-like emitters that require footprints many times wider than the needle tips. Thus, this approach will likely be confined to low thrust, albeit important, applications.

The nanoFET can also be contrasted with the colloid thruster that forms and accelerates small liquid droplets. $^{7} \quad$ It is known that with the right emission current and temperature, charge extraction in FEEPs can produce instabilities that result in the formation of charged microscopic droplets (colloids). While these droplets could, in principle, be used to accomplish the same goal as our nanoparticles, it is difficult to control their size and a distribution is expected. Our nanoFET propulsion concept avoids the conditions that generate colloids and allows us to tune the nanoparticle size independently of other factors.

Unlike colloid thrusters, nanoFET emits charged conducting nanoparticles of well-defined size and charge-to-mass ratios. Figure 3 depicts a model of a single emitter from the nanoparticle thruster using an insulating liquid reservoir and cylindrically shaped particles. These nanoparticles would be transported to a small liquid-filled reservoir by a micro-fluidic flow transport system. Particles that come into contact with the bottom conducting plate would become charged and pulled to the liquid surface by the imposed electric field. If the electrostatic force near the surface can cause charged nanoparticles to break through the surface tension, field focusing would quickly accelerate the particles through the surface. Once extracted, the charged nanoparticles would be accelerated by the vacuum electric field and ejected. It is also possible to consider a similar configuration but with a slightly conducting liquid where nanoparticles arrive at the surface and are preferentially charged relative to the surrounding surface, thus resulting in extraction.

Note that neutralization of the charged particles is required. However, the concept of using MEMS-based fabrication technology to manufacture arrays of small vias with biased grids (just like 


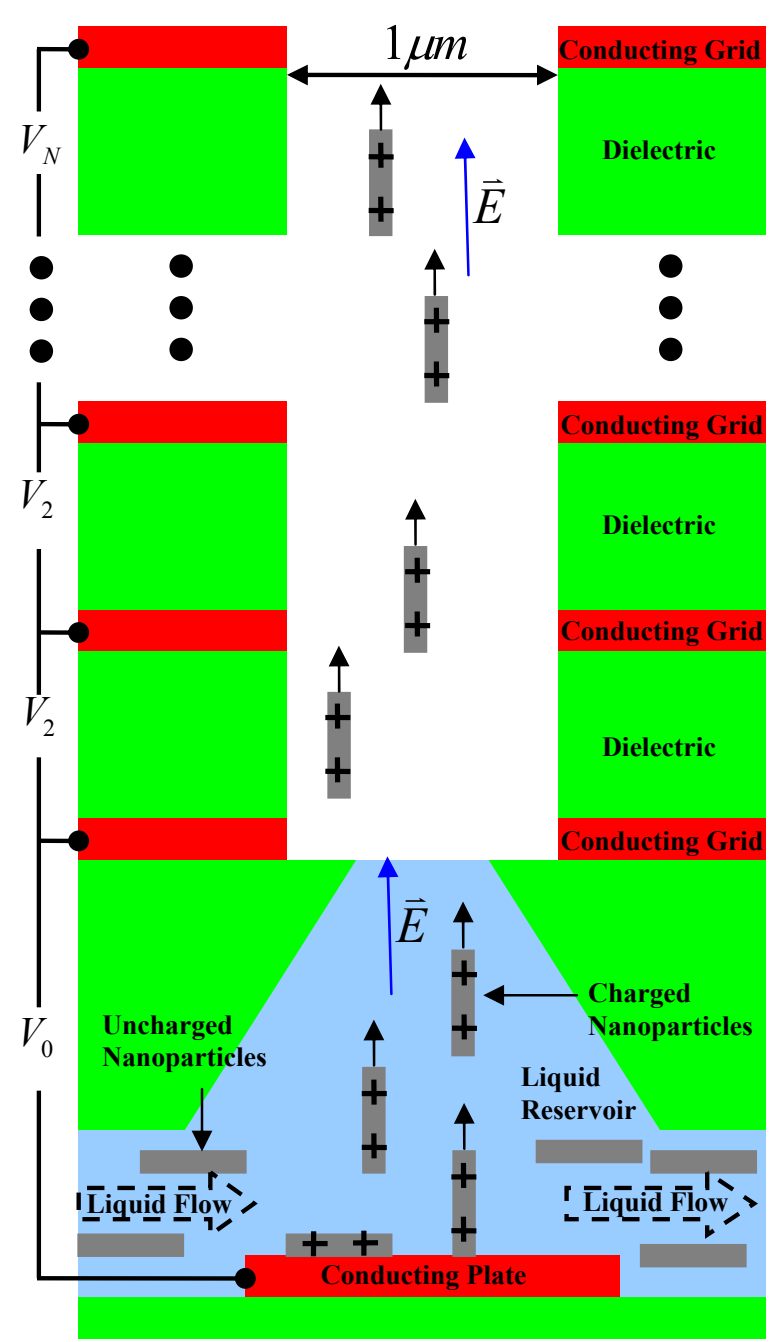

Figure 3: Structure of a single emitter, multi-grid nanoparticle thruster using an insulating liquid.
nanoFET) to extract electrons has been discussed previously, ${ }^{8,9}$ called here the Field Emission Array Cathode (FEAC). Further, a more attractive option may be to bias separate sections of the grid with opposite polarity to simultaneously emit both positive and negatively charged nanoparticles or oscillating the polarity of the entire thruster positive and negative, eliminating the need for a separate neutralizer system.

This paper focuses mostly on initial experimental test results and briefly discusses the system advantages of nanoFET. The theoretical aspects are discussed in a parallel paper, AIAA$2006-4335 .^{10}$

\section{Advantages of nanoFET}

The nanoFET concept derives its benefits from the (1) the ability to operate at very high efficiency; (2) adjust specific impulse over a large range (possibly covering $\sim 100 \mathrm{~s}$ to $\sim 10,000 \mathrm{~s}$ ); (3) scalability over a large power or thrust range; and (4) the elimination of lifelimiting factors common in state-of-the art ion thrusters. We expand on these advantages below:

\section{A. Highly Integrated System}

The use of MEMS technology enables a "flat panel" thruster design that incorporates power processing as well as nanoparticle manufacture, storage, feed, extraction, and acceleration. Such compact design simplifies propulsion system integration and lowers thruster specific mass. Because different regions of nanoFET can emit particles of opposite polarity, neutralizer requirements are simplified as nanoFET is a self-neutralizing thruster.

\section{B. Geometrically Scalable}

The use of flat panel MEMS technology enables a single thruster type, which can be straightforwardly scaled, to span a broad thrust range. This plug-and-play technology decouples thruster design from spacecraft design. As such, by providing better overall performance and a wider range of operating parameters, thus enabling a broader set of mission modes, nanoFET is both mission enhancing and mission enabling.

\section{Eliminates Primary Life Limiting Factors of other EP Systems}

Charging of the nanoparticles is accomplished without ionization, meaning greater reliability and the absence of cathodes and charge-exchange collisions that are the principal lifetime limiters of current electric propulsion systems. Propellant charging, as opposed to propellant ionizing, also accounts for part of the efficiency gains nanoFET affords.

\section{Improved Controllability}

Charge-to-mass ratio is much better regulated for nanoparticles than for colloids, resulting in better controllability for nanoFET versus colloidal thrusters. 


\section{E. High Thrust Density}

Whereas state-of-the-art ion thrusters operate substantially below the space charge limit to ensure proper ion optics operation, nanoFET should operate much closer to the space charge limit since the charges will be contained in a fewer number of particles. Thus, thruster specific mass is reduced.

\section{F. Enormous Specific Impulse Range}

Charging nanoparticles versus ionizing atoms means that the charge-to-mass ratio can be adjusted over a huge range even within a single mission and with a more efficient expenditure of energy. It also increases mission flexibility. For example, nanoFET would be able to accomplish an orbit transfer with different thrusting times dependent on $I_{\mathrm{sp}}$ level, while a constant $I_{\mathrm{sp}}$ thruster can only perform the same transfer with a single thrusting time. If it is important to achieve the transfer in a short time, nanoFET can operate in a low $I_{\mathrm{sp}}$ and high thrust mode. On the other hand, if thrusting time is not important, then nanoFET can operate at a high $I_{\mathrm{sp}}$ and conserve propellant. A highly variable $I_{\mathrm{sp}}$ system provides many more options and more mission flexibility than a constant $I_{\mathrm{sp}}$ system. This added flexibility could be important in off-nominal scenarios that would extend the possible range of safe operation or increase abort scenarios for given missions.

The ability to tune propulsion characteristics via different nanoparticle dimensions and charge states should permit thrust efficiencies over $90 \%$ for a specific impulse range of $100 \mathrm{~s}$ to $10,000 \mathrm{~s}$. Such high efficiencies result in thrust-to-power ratios, especially at low specific impulse, that are orders of magnitude greater than state-of-the-art ion and Hall thrusters. Consequently, nanoFET could operate at high specific impulse in cruise mode and yet switch to a high thrust and low $I_{\mathrm{sp}}$ mode when needed. This flexibility provides a wider margin for mission designers to accommodate off-nominal mission scenarios as well as dynamic retasking of space assets to take advantage of inflight opportunities.

For example, by using just three types of nanoparticles, the nanoFET system can span an $I_{\mathrm{sp}}$ range from $100 \mathrm{~s}$ to $10,000 \mathrm{~s}$. Three possible carbon nanotube particles are listed in Table 1.

Note that the entire $I_{\mathrm{sp}}$ range could potentially be covered with over $90 \%$ thrust efficiency using just the three particle types as shown in Figure $4 .{ }^{11}$ Such performance can be obtained at reasonable voltages between 800 to $10,000 \mathrm{~V}$ due to the MEMS gated structures that provide the extraction and acceleration electric fields. Note that in the nanoFET system, efficiency losses may be due to viscous drag in the liquid, charge loss to the liquid, particle impingement on the gates, and beam defocusing.

NanoFET's thrust-to-power performance compared to other state-of-the-art thruster types is shown in Figure 5. This level of performance could provide mission designers with the flexibility to engage a high thrust mode to climb out of gravity wells, perform abort scenarios or emergency maneuvers, and reduce trip times.

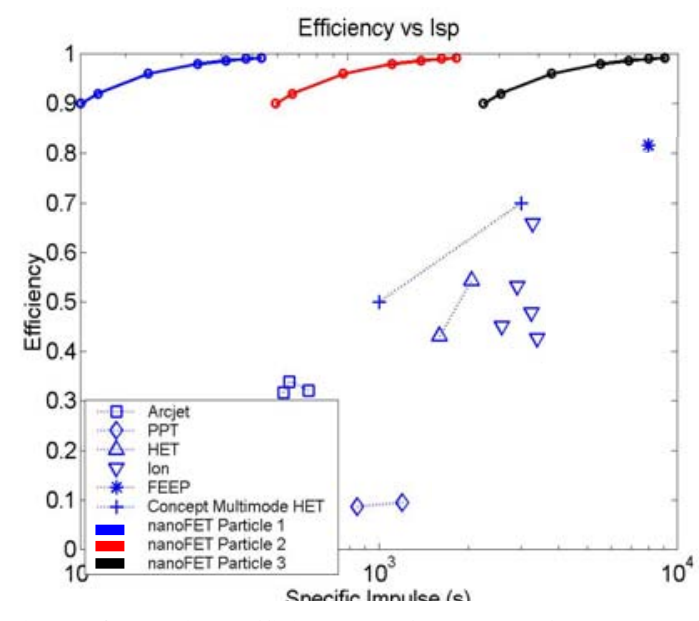

Figure 4: Possible efficiency performance of nanoFET for entire specific impulse range compared with other electric propulsion systems. The nanoparticles are specified in Table 1.

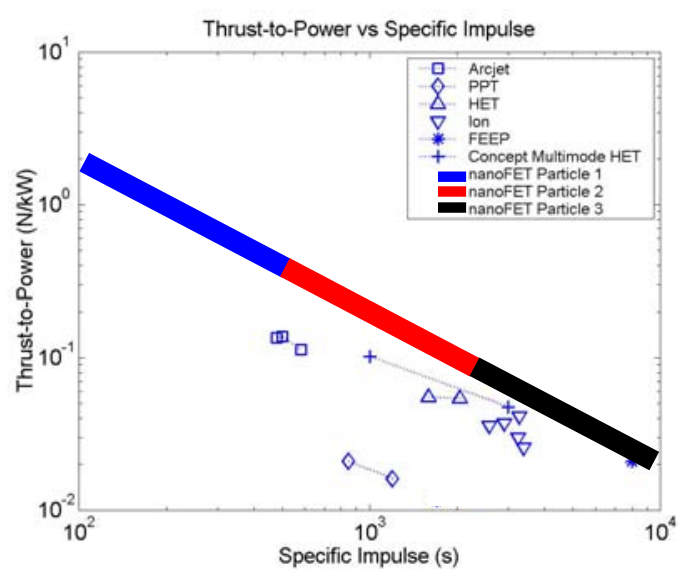

Figure 5: High thrust-to-power ratio for nanoFET compared to other electric propulsion systems. The nanoparticles are specified in Table 1 . The thick blue-redblack line represents $90 \%$ to $100 \%$ efficiency. 


\section{Experimental Study of nanoFET}

The nanoFET system charges conductive particles in contact with an electrode that is immersed in a dielectric liquid. When the particles acquire sufficient charge, they are lifted off the electrode by an imposed electric field and transported to the liquid surface. There, the electrostatic force acting on the particles must overcome the surface tension force to extract the particles from the liquid. Following extraction, the particles are accelerated by the imposed electric field to provide thrust. To understand nanoFET's governing physics, the following aspects must be understood:

- particle charging and lift-off;

- particle dynamics within the liquid;

- particle extraction through the liquid surface; and

- liquid surface instabilities

\section{A. Particle Charging and Transport Experiments}

We have experimentally demonstrated the ability to charge and transport conducting particles submersed in an insulating liquid with intense electric fields using "scaled-up" particles. Figure 6 shows a diagram of our experimental setup. The setup includes a liquid-filled electrode gap with conducting particles initially resting on the bottom electrode and a highvoltage power supply used to generate electric fields in the gap between the electrodes.

The $10 \mathrm{~cm}$ diameter circular electrodes are made of stainless steel. We used silicone oil as our

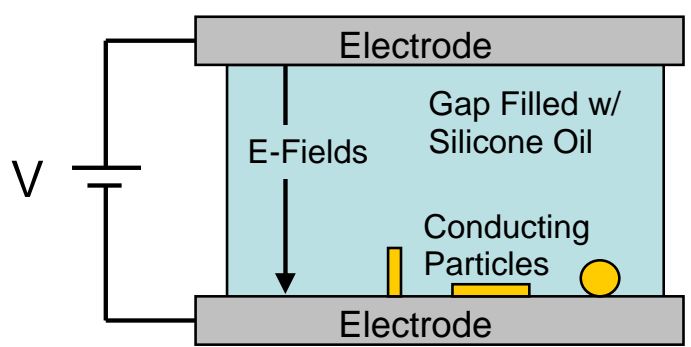

Figure 6: Experimental setup for particle oscillation. liquid because of its low conductivity, high breakdown potential, and very low vapor pressure. Silicone oil has a density of $965 \mathrm{~kg} / \mathrm{m}^{3}$, a viscosity of $100 \mathrm{cSt}$, and a conductivity of $3.33 \times 10^{-13} \mathrm{~S} / \mathrm{m}$. The particles (Figure 7) were mostly millimeter-sized spheres and cylinders made from aluminum because of its high conductivity and low density.

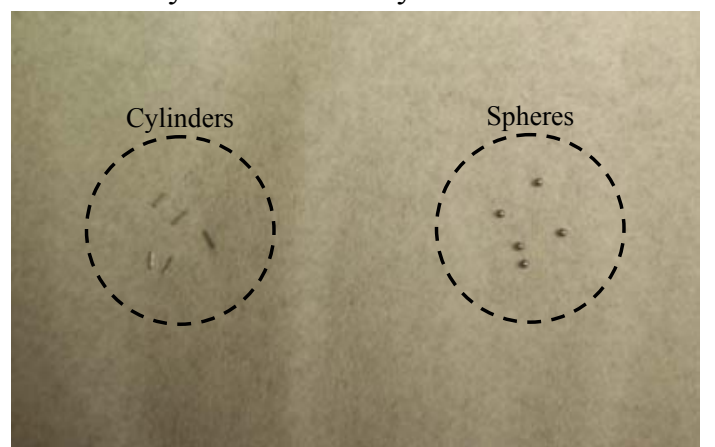

Figure 7: Picture of millimeter-sized aluminum spherical and cylindrical particles used in experiments. Spheres have an 800- $\mu \mathrm{m}$ diameter. Cylinders have a $300-\mu \mathrm{m}$ diameter and $2.5-\mathrm{mm}$ length.

During particle transport, four forces act on the particles: electrostatic, gravitational, buoyant, and drag. Figure 8 shows these forces on a particle being transported upward.

To begin the experiment, the voltage between the electrodes was zeroed with the positive lead connected to the upper electrode and the ground connected to the bottom electrode. The particles were placed at rest on the bottom electrode. As the voltage was increased to generate uniform electric fields in the gap, the conducting particles in contact with the bottom electrode began to charge negatively along with the bottom electrode. The charge acquired by 
conducting particles submersed in a liquid, in contact with an electrode, and subjected to an electric field was determined by Félici. ${ }^{12,13,14,15}$ The charges acquired by spherical and vertically oriented cylindrical particles, respectively, are given as follows:

$$
q_{0, \mathrm{sp}}=\frac{2 \pi^{3}}{3} r^{2} \varepsilon_{\ell} E_{\ell},(1) \quad q_{0, \mathrm{cy}-\mathrm{v}}=\pi \frac{l^{2}}{\ln \left(\frac{2 l}{r}\right)-1} \varepsilon_{\ell} E_{\ell} .
$$

Once the electrostatic force on the charged particles was great enough to overcome the image charge, surface adhesion, and gravitational forces, they were lifted from the bottom electrode and transported to the upper electrode. Upon reaching the positively charged upper electrode, the particles underwent a charge exchange, becoming positively charged with the same magnitude as when they were charged on the bottom electrode. The particles were then transported back down through the liquid to the bottom electrode. The resulting particle oscillation is depicted in Figure 9.

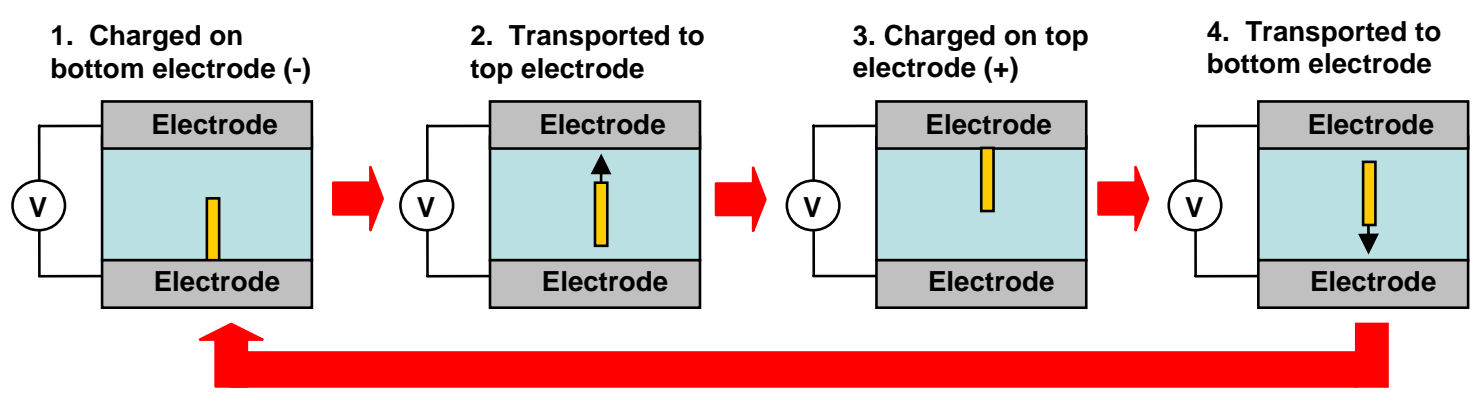

Figure 9: Stages of particle oscillation experiment.

From our experiments, we found that we could put both spherical and cylindrical particles into oscillation using electric fields. As expected, cylindrical particles were able to oscillate at much lower electric fields than the spherical particles due to their greater geometrical aspect ratio providing greater field focusing at the cylinder ends.

Most of the experiments were performed with aluminum spheres of $800 \mu \mathrm{m}$ and $1600 \mu \mathrm{m}$ diameters or aluminum cylindrical particles with diameters of 300 $\mu \mathrm{m}$ and lengths ranging from $1 \mathrm{~mm}$ to $2.5 \mathrm{~mm}$ initially placed horizontally on the bottom electrode. When the voltage between the electrodes was increased, the horizontal cylinders were pulled into a vertical position before oscillating. Once the particles were lifted from the electrode and set into motion, they reached their terminal velocity very quickly, where the terminal velocity could be controlled readily by adjusting the potential applied between the electrodes.

Figure 10 shows a picture of two particles in mid-oscillation between the electrodes. This experiment used $300 \mu \mathrm{m}$ diameter by $1.5 \mathrm{~mm}$ long aluminum cylindrical particles, a $12.7 \mathrm{~mm}$ gap between

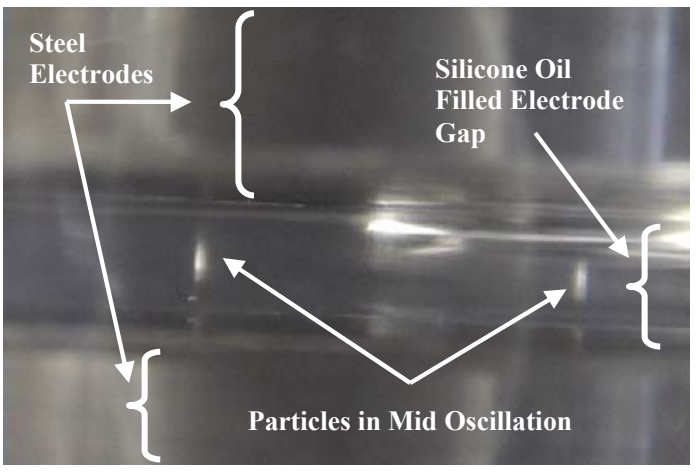

Figure 10: Two cylindrical particle in oscillation. The particles are aluminum with dimensions: $\mathbf{d}=\mathbf{3 0 0}$ $\mu \mathrm{m}, \mathrm{l}=\mathbf{1 . 5} \mathrm{mm}$. Electrode gap is $\mathbf{1 2 . 7} \mathrm{mm}$. the electrodes, and silicone oil filling the gap.

From the particle oscillation experiment, we learned that we can charge and transport conducting particles that are submersed in an insulating liquid by controlling the electric field around the particles. Initial experiments suggest that particle charging agrees with Félici's predictions.

\section{B. Particle Extraction Experiments}

The next step is to establish the feasibility of extracting particles through a liquid surface. Figure 11 shows the experimental setup used for the particle extraction experiment. This setup is very similar to the particle oscillation setup, but now the liquid only partially fills the electrode gap, leaving the upper 


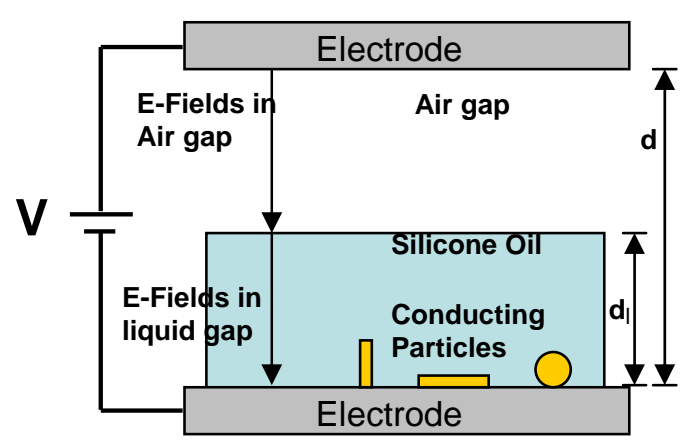

Figure 11: Experimental setup for particle extraction.

section of the gap air-filled.

This experiment was run in a very similar manner as the particle oscillation experiment. The voltage between the electrodes was initially zeroed with the positive lead connected to the upper electrode and the ground connected to the bottom electrode. The cylindrical or spherical particles were placed at rest on the bottom electrode. When the voltage was increased, generating an electric field in the gap, the conducting particles were charged. The electric force on the charged particles lifted and transported them to the liquid-air interface. There, if the electric force on the particles was strong enough to overcome the additional surface tension force, they would break through the surface tension and particle extraction would be achieved. After being extracted, they would travel through the air gap and contact the upper electrode, where they underwent a charge exchange and were accelerated back into the liquid and down to the bottom electrode. The resulting particle oscillation is depicted schematically in Figure 12.

1. Charged on bottom electrode (-)
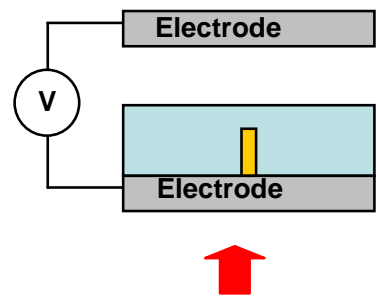

6. Pulled through liquid surface

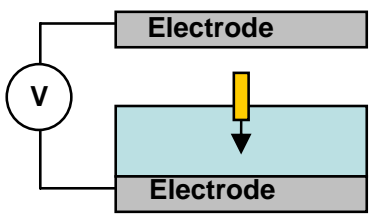

2. Transported to liquid surface
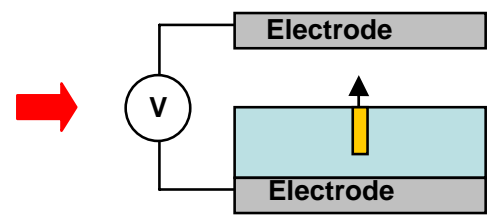

5. Transported to liquid surface

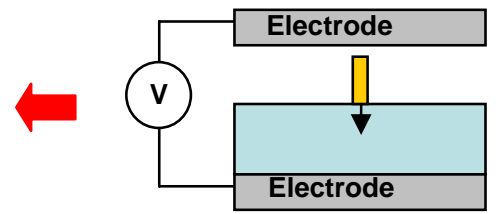

3. Extracted through liquid surface
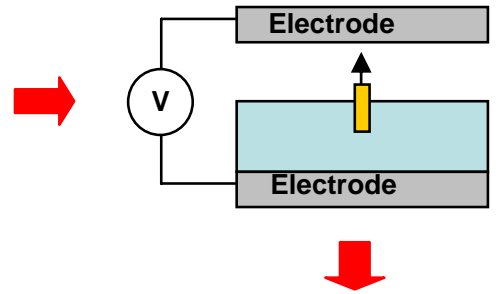

4. Charged on top electrode (+)

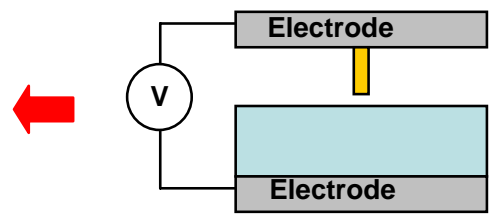

Figure 12: Stages of particle extraction

From our experiment, we established the feasibility of extracting particles from a liquid into air. In addition, we also measured the minimum required electric fields to extract particles of various sizes and shapes for different liquid heights and found very good agreement with our theoretical predictions. Just as in the oscillation experiment, the cylindrical particles were extracted at much lower electric fields than the spherical particles due to the greater field focusing at the tips. Moreover, increasing the aspect ratio of the cylindrical particles lowered the required electric field for the same reason. Results from these experiments are discussed in Section C along with Taylor Cone formation.

Figure 13 shows a series of pictures of two particles being extracted. This experiment used 300 $\mu \mathrm{m}$ by $1.5 \mathrm{~mm}$ aluminum cylindrical particles, a $12.7 \mathrm{~mm}$ gap between the electrodes, and silicone oil filling the gap up to $6 \mathrm{~mm}$. Picture (a) shows the two particles on the bottom electrode oriented vertically. Next, the particles are pulled to the liquid surface as seen in picture (b). Finally the particles are extracted through the liquid surface and are in the air gap in picture (c). It is difficult to see the particles in the final picture because they are moving very quickly.

From the particle extraction experiment, we proved that it is feasible to extract particles from liquid using electric fields, an important step to demonstrating nanoFET's feasibility. 

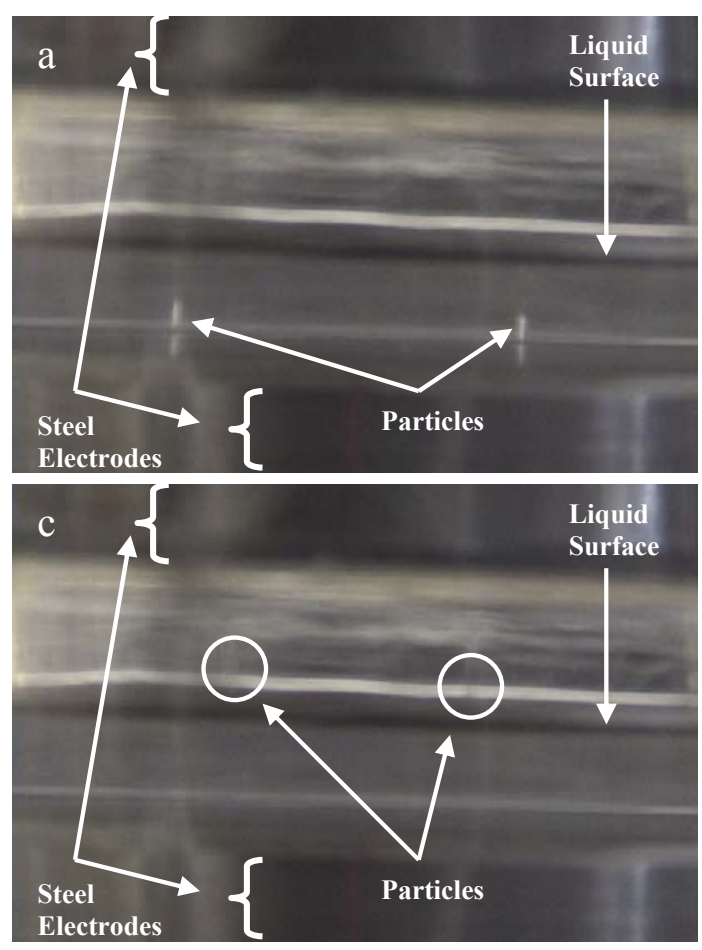

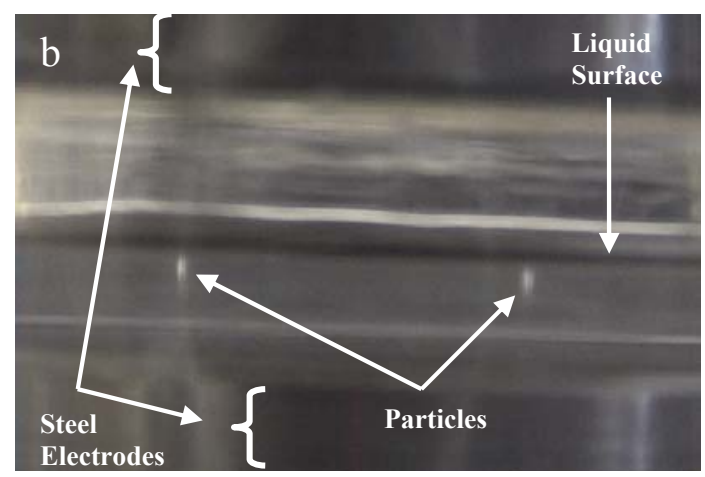

Figure 13: Two cylindrical particle being extracted. The particles are aluminum with dimensions: $d=\mathbf{3 0 0}$ $\mu \mathrm{m}, \mathrm{l}=1.5 \mathrm{~mm}$. Electrode gap is $12.7 \mathrm{~mm}$.
a) particles are on bottom electrode b) particles pulled to liquid surface c) particles extracted and in air gap (difficult to see particles because of high velocities)

\section{Surface Instability and Taylor Cone Formation Experiments}

While running the particle extraction experiments, we found that at very high electric fields, Taylor cones would form on the surface of the liquid. Taylor cones are a result of instabilities in the liquid surface and form when the electric field pulls on free charge at the liquid surface. At very high electric fields, small charged liquid droplets can be pulled off of the liquid surface just as in a colloid thruster. Figure 14 shows the stages of Taylor cone formation.

\section{Charge is pulled to} surface

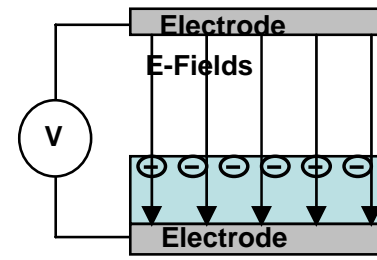

2. E-field pulls liquid up, surface tension pulls down

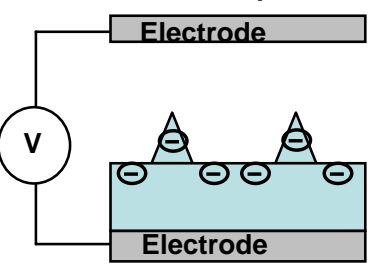

3. Charged droplets are pulled off surface

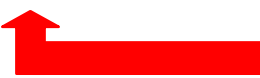

Figure 14: Stages of Taylor cone formation.

We believe it will be important for nanoFET to operate in a regime where Taylor cones do not form. In other words, we need to be sure that it is possible to extract particles through a liquid surface at electric field strengths below those that cause the liquid to become unstable. The minimum vacuum (air) gap electric field that would cause a perturbed fluid surface to become unstable is given by a corrected version of Tonks's ${ }^{16}$ formula adapted for dielectric liquids,

$$
E_{0, \min }=\left(\frac{4 g \gamma \rho_{\ell}}{\varepsilon_{0}{ }^{2}}\right)^{\frac{1}{4}}\left[1+\left(\frac{\varepsilon_{0}}{\varepsilon_{\ell}}\right)^{2}\right]^{-\frac{1}{2}} \text {. }
$$

This experiment was run in a very similar manner as the particle extraction experiment, but without the particles. The electric field in the gap was increased until Taylor cones began to form. The electric fields required to form the cones were determined as a function of the liquid height and compared 
extremely well with theory.

Next, we compared the electric fields required to extract various particles through the liquid surface with the minimum fields at which cones begin to form. Figure 15 shows a plot of the required fields to extract different particles and the electric fields necessary to form cones as a function of liquid height. The total electrode gap was $12.7 \mathrm{~mm}$. The spherical particles were $800 \mu \mathrm{m}$ in diameter and the cylindrical particles were $300 \mu \mathrm{m}$ in diameter and $1.5 \mathrm{~mm}$ in length. Both particles were aluminum. We found that the average extraction electric field was $1.8 \times 10^{6} \mathrm{~V} / \mathrm{m}$ for spherical particles and $1.3 \times 10^{6} \mathrm{~V} / \mathrm{m}$ for cylindrical particles; these values are within $20 \%$ of the theoretical predictions using a

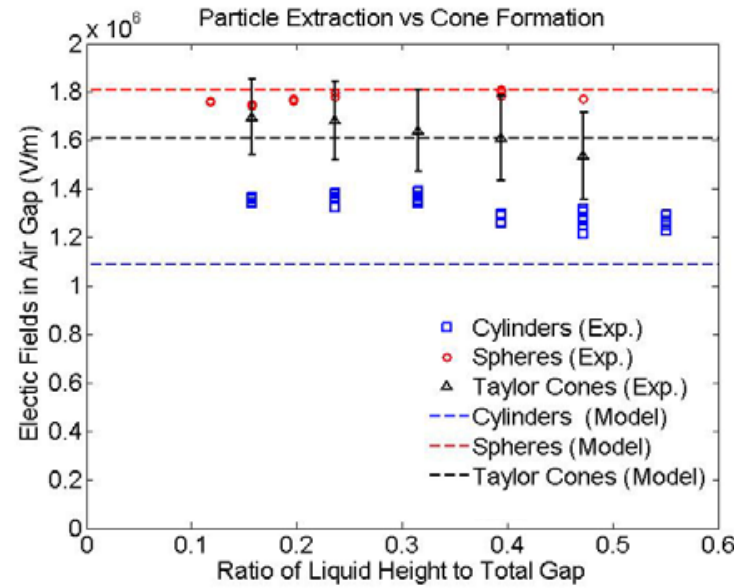

Figure 15: Required electric fields for particle extraction and Taylor cone formation. preliminary particle extraction model. For Taylor cone formation, the measured formation electric field of $1.6 \times 10^{6} \mathrm{~V} / \mathrm{m}$ agreed very well with Equation 3. The experiments indicate that a regime exists where particles may be extracted prior to the onset of liquid instability, and will be discussed in the next section. It is also important to note that the presence of particles does not reduce the critical threshold for Taylor cone formation.

\section{Feasible Design Space}

The feasible design space for the proof-of-concept test configuration using cylindrical particles is shown in Figure 16. For nanoFET to be feasible, emitter configurations must exist such that the electric fields in the liquid are greater than the minimum electric fields needed to lift off the particles from the electrode surface. At the same time, the corresponding vacuum electric fields must be greater than the minimum electric fields needed to extract the particle from the liquid surface while less than the minimum electric fields that would cause liquid surface instabilities.

Note that the theory indicates the existence of operational regimes where particles may be extracted with lower electric fields than required for Taylor cone formation, a finding that was validated by experiment. Test data indicate excellent agreement between the predicted and measured electric field strength needed to cause liquid surface instability. Use of a simplified particle extraction model led to a difference from experimental results for the extraction fields by less than $20 \%$. The need to acquire a better understanding of the particle field enhancement effect at the liquid surface as well as the effects of surface tension on particle extraction will be investigated in the future. Continued exploration of the feasible design space as particles are scaled

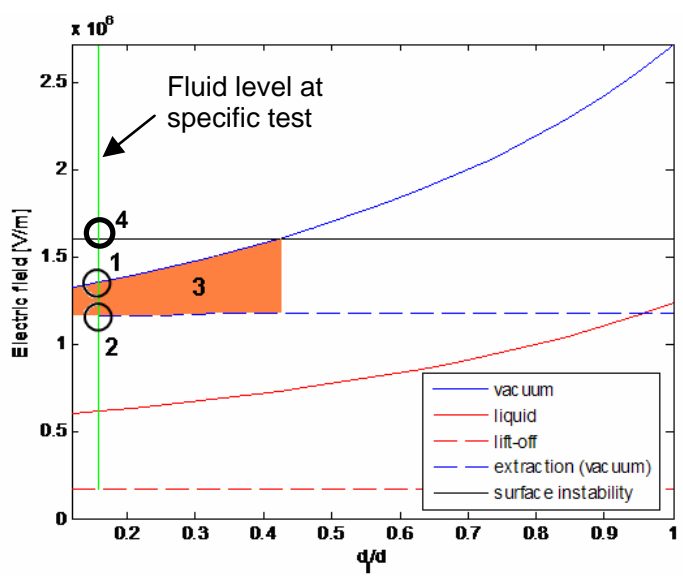

Figure 16: The feasible design space (3) for the proofof-concept test configuration using cylindrical particles. Particle extraction threshold for experiment (1) and theory (2); Taylor cone formation threshold (4) for experiment and theory. down to the nano-scales is in progress.

\section{E. Particle Extraction through a Grid}

The next step in the experimental process was to demonstrate the feasibility of extracting particles through a grid structure, since the nanoFET design calls for a multi-grid structure for particle extraction and acceleration. This experiment was another extension to the particle extraction experiment where a grid structure was inserted in the air gap as shown in Figure 17. The holes in the grid structure were designed to have a diameter approximately equal to the gap dimension in order to keep the electric fields uniform. 
The power supply connected from the bottom electrode to the grid structure was responsible for particle charging, extraction, and acceleration through the grid. The power supply connected from the grid to the upper electrode was responsible for continuing to accelerate the particles.

This experiment was performed several times with various particle shape and sizes, and it was experimentally proven that particle extraction through a grid structure is feasible. Figure 18 shows a series of pictures showing spherical particles being extracted through a grid structure. Picture (a) shows nine particles resting on the bottom electrode before the electric field was increased, and picture (b) shows the particles stuck to the upper electrode after being

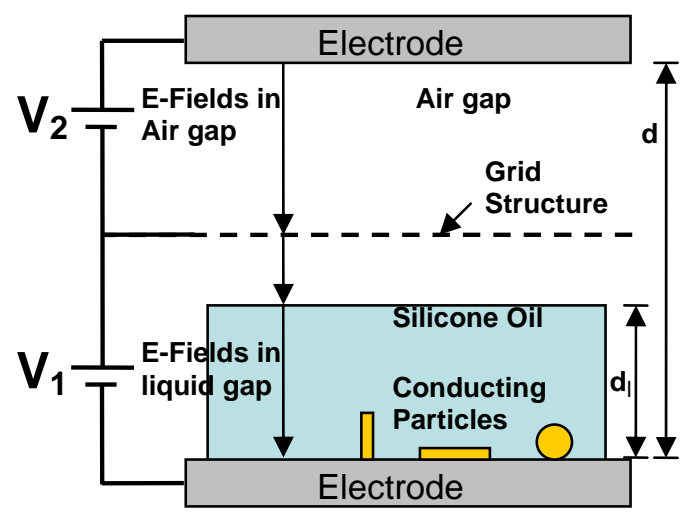

Figure 17: Experimental setup for particle extraction through a grid structure.
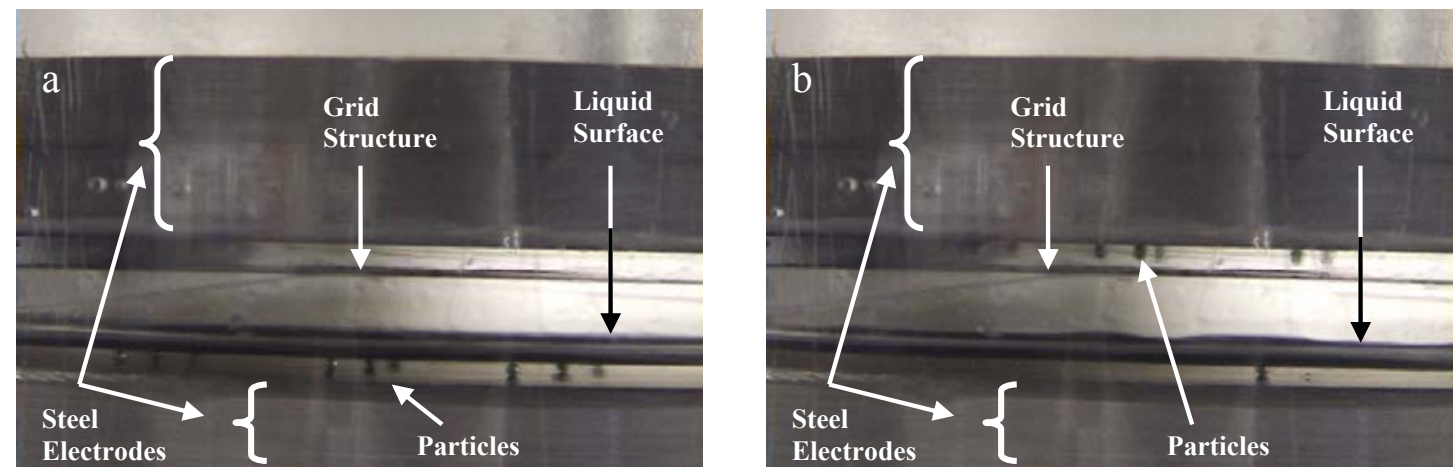

Figure 18: Spherical particle extraction through a grid structure.

a) Particles are on bottom electrode before electric field is turned on.

b) Particles are stuck to upper electrode after being extracted through grid structure.

\section{F. Vacuum Experiments}

Since the nanoFET will be operating in space it is important to run the experiments discussed earlier in vacuum to be assured that particle extraction and cone formation still behave as expected.

A series of experiments have been run in a vacuum environment to demonstrate that the nanoFET concept works in vacuum conditions. Preliminary results suggest that particle extraction and the onset of liquid surface instability are not strongly dependent on the external pressure. We plan to continue these experiments in the near future.

\section{G. Experiments with Nanoparticles}

To experimentally verify that nanoparticles can be charged and transported just like the millimetersized particles, we have begun experiments using various spherical nanoparticles ranging in size from 5 $\mathrm{nm}$ to $70 \mathrm{~nm}$. The materials that we are currently working with are silver, nickel, and copper.

We have shown that the nanoparticles can be charged and transported when submersed in a liquid

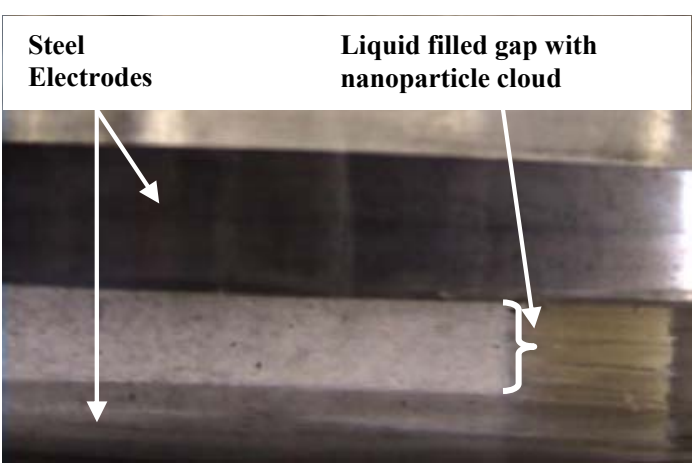

Figure 19: Silver nanoparticle cloud oscillating between electrodes when submersed in silicone oil. 
with electric fields just like the larger particles. We used the same experimental setup as in the particle oscillation experiment (Figure 6). Figure 19 shows a picture of a silver nanoparticle cloud put into oscillation within an electrode gap filled with silicone oil. It is important to note that the larger visible distinguishable particles are clumps of nano-spheres, since the individual nanoparticles are not visible with the naked eye.

\section{Conclusions}

We have addressed several fundamental questions regarding nanoFET operation with "scaled-up" particles. We have demonstrated the transport of conducting particles through an insulating liquid by way of an electric field and have achieved particle extraction from a liquid prior to the onset of liquid surface instability. In addition, particle extraction through a gridded structure was accomplished, and vacuum and nanoparticle tests are in progress. These experimental results have validated our theoretical models and represent a significant step towards proving the fundamental feasibility of nanoFET.

\section{Acknowledgements}

This project is funded by a Phase 1 grant from the NASA Institute for Advanced Concepts (NIAC) and supported by a National Defense Science and Engineering Graduate Fellowship. Thanks to Matthew Forsyth for helping to construct the experimental setup and Robb Gillespie for building our vacuum chamber. Special thanks to Bailo Ngom from the University of Michigan's Plasmadynamics and Electric Propulsion Laboratory for Figure 1.

\section{References:}

1 Musinski, L., Liu, T., Gilchrist, B., Gallimore, A., Keidar. M., "Scalable Flat-Panel MEMS/NEMS Thruster," IEPC-2005-176, Proceedings of the International Electric Propulsion Conference, Princeton, New Jersey, October 31 - November 4, 2005.

2 Behan, Niall, "Nanomedicin and Drug delivery at the University of Limerick," The University of Limerick. http://www.ul.ie/elements/Issue4/behan.htm

3 Chesta, E., Nicolini, D., Robertson, D. and Saccoccia, G., "Experimental Studies Related to Field Emission Thruster Operation: Emission Impact On Solar Cell Performances And Neutralization Electron Backstreaming Phenomena," IEPC-2003-102, Proceedings of the International Electric Propulsion Conference, Toulouse, France, March 17-20, 2003.

4 Marcuccio, S. "Attitude and Orbit Control of Small Satellites and Constellations with FEEP Thrusters," Electric Rocket Propulsion Society, 1997.

5 Marcuccio, S. "FEEP Microthruster Technology Status and Potential Applications," International Astronautical Federation, 1997.

6 Marcuccio, S. "FEEP Thrusters," Nov. 1998, http://www.centrospazio.cpr.it/Centrospazio6FEEP.html.

7 Stark, John. "Micro-Fabrication and Operation Nano Emitters Suitable for a Colloid Thruster Array," University of London, UK, https://escies.org/public/mnt4/S9.1Stark.pdf

8 Morris, D.P., Gilchrist, B.E., Gallimore, A.D., "Application of Dual Grids to Cold Cathode/ Field Effect Electron Emission," AIAA-2005-3669, 41 st Joint Propulsion Conference, Tucson, AZ, July 1013, 2005.

9 Goldberg, H., Encarnación, P., A., Morris, D., Gilchrist, B., Clarke, R., "Cold-Cathode Electron Field Emission of Boron Nitride Thin Film with a MEMS-Based Gate for Space Applications, AIAA-20043499, 40th Joint Propulsion Conference, Ft. Lauderdale, FL, July 11-14, 2004.

10 Liu, T., Keidar, M., Musinski, L., Gallimore, A., Gilchrist, B., "Theoretical Aspects of Nanoparticle Electric Propulsion.” AIAA-2006-4335, 42nd Joint Propulsion Conference, Sacramento, CA, July 912, 2006.

11 Wertz, Larson, "Space Mission Analysis and Design $3^{\text {rd }}$ Ed." 1999.

12 Felici, N. Rev. Gen. Elect., 75, pp. 1145-1160, 1966

13 Tobazeon, R. "Behavior of Spherical and Cylindrical Particles in an Insulating Liquid Subjected to a DC Uniform Field." Laboratoire d'Electrostatique et de Materiaux Dielectriques. BP 166-38042 Grenoble Cedex 9 (France) pp. 415-420.

14 Khayari, A. "The Charge Acquired by a Spherical Ball Bouncing on an Electrode: Comparison Between Theory and Experiment;" 2000 Conference on Electrical Insulation and Dielectric Phenomena. 
15 Choi, Changrag. "Dynamic Motion of a Conductive Particle in Viscous Fluid Under DC Electric Field," IEEE Transactions on industry Applications, vol. 37, No. 3, May/June $2001{ }^{\text {Error! Reference source not }}$ found.

16 Tonks, L., "A Theory of Liquid Surface Rupture by a Uniform Electric Field," Physical Review, Vol. 48, 15 September 1935, pp. 562-8.

17 Calero, J. "The electrohydrostatics of a Conductive Liquid Meniscus," IEEE 1988, p 1547-1551.

18 Chock, R. "Photovoltaic \& Space Environment Branch," NASA Glenn Research Center, July 2002, http://powerweb.grc.nasa.gov/pvsee/publications/tropix/Paper/AppA.html.

19 J.R. Brophy, J.E. Polk, V.K. Rawlin, Ion engine service life validation by analysis and testing, AIAA Paper 96-2715, July 1996.

18 M. Fehringer, F. Ruedenauer, W. Steiger, ESTEC Contract 12376/97/NL/PA Tech. Note No. 2, 1997.

19 Najafi, Khalil. Personal Communications, University of Michigan, 2004.

20 Smith, R.C., Carey, J.D., Forrest, R.D., Silva, S.R.P., "Effect of aspect ratio and anode location on the field emission properties of a single tip based emitter," J. Vac. Sci. Technol. B 23(2), Mar/Apr 2005, pp. 632-5. 